\title{
Retrospective Evaluation of Acute Kidney Injury: Extended Infusion versus Standard Infusion Piperacillin/Tazobactam with Vancomycin
}

\author{
Deanna Ratermann, Michael Sepulveda and Dan Meier \\ St. Elizabeth Healthcare Department of Pharmacy, Edgewood 41017, Kentucky, United States
}

\begin{abstract}
Antibiotics cause 36 percent of all cases of drug-induced AKI (acute kidney injury). Research suggests that drug-induced AKI increases hospital length of stay, ventilator days and mortality. The purpose of this study was to determine which administration method of piperacillin/tazobactam (pip/tazo) would have a higher incidence of AKI in combination with vancomycin. All patients greater than 18 years old admitted to the general medical floor, who were receiving combination therapy were included in the study. Excluded patients comprised: patients with a baseline SCr (serum creatinine) greater than $2 \mathrm{mg} / \mathrm{dL}$, receiving peritoneal dialysis or hemodialysis, an ICU stay, pregnancy, and concomitant use of defined nephrotoxic agents. AKI was defined as an absolute increase in $\mathrm{SCr}$ of $0.5 \mathrm{mg} / \mathrm{dL}$ or a $50 \%$ increase from baseline. The primary outcome of AKI occurred in 18 out of 63 patients (28.6\%) in the standard-infusion pip/tazo plus vancomycin group and 9 out of 35 patients $(25.7 \%)$ in the extended-infusion pip/tazo plus vancomycin group $(Z=-0.30, p$-value $=0.381)$. AKI is very prevalent with one of our most frequently used empiric antibiotic combinations. While this study found no significant difference, as pharmacists, we learned it is still necessary to closely monitor patients' receiving this antibiotic combination.
\end{abstract}

Key words: Piperacillin/tazobactam, vancomycin, acute, kidney, injury.

\section{Introduction}

Medical literature has reported more than 35 definitions of acute kidney injury. Prior to 2004, AKI was known as acute renal failure. This terminology was changed to account for the varying degrees of kidney injury or damage that can occur without causing complete renal failure [1,2]. The most accepted definition of AKI in today's literature is an abrupt loss of kidney function, causing build-up of nitrogenous waste and a deregulation of extracellular volume and electrolytes [1,2]. Objectively, AKI is often defined as an increase in $\mathrm{SCr}$ by $0.5 \mathrm{mg} / \mathrm{dL}$ or a 50 percent increase from baseline. Drug-induced AKI occurs in approximately 25 percent of all hospitalized patients. Of that number, up to 36 percent of cases have been linked specifically to antibiotics [2, 3]. Research

Corresponding author: Deanna Ratermann, Pharm.D., research field: internal medicine. E-mail: Deanna.Ratermann@stelizabeth.com. suggests that drug-induced AKI increases hospital length of stay, ventilator days, and mortality. In addition, AKI could lead to permanent kidney disease, causing patients to require renal replacement therapy such as peritoneal dialysis or hemodialysis [1-3].

Vancomycin and piperacillin/tazobactam (pip/tazo) are frequently used in combination as empiric broad-spectrum antibiotic therapy for suspected severe infections. According to several studies in medical literature, the incidence of $\mathrm{AKI}$ is increased in patients treated with combination vancomycin and pip/tazo therapy versus monotherapy of either agent [4-6]. There are several possible mechanisms for drug-induced AKI. Researchers hypothesize vancomycin causes a dose dependent cellular degeneration of the proximal and distal tubule basement membranes in the kidney. This mechanism of drug-induced AKI is known as acute tubular necrosis $[7,8]$. Similar to other beta-lactam antibiotics, pip/tazo 
causes AKI by a non-dose dependent mechanism known as acute interstitial nephritis, which is characterized by a hypersensitivity reaction leading to inflammation and damage of the kidney interstitium [9, $10]$.

Recently healthcare institutions began transitioning from 30 minutes SI (standard-infusion) pip/tazo to 4 hours EI (extended-infusion) pip/tazo. All beta-lactams, including pip/tazo, exhibit time dependent bacterial killing. Optimal bactericidal activity is observed when time above the MIC (minimum inhibitory concentration) is maximized. Several studies have focused on optimizing clinical use of pip/tazo by increasing the duration of exposure through extended-infusion formulations. EI pip/tazo dosed at 3.375 grams every eight hours allows for greater than 90\% MIC target attainment at a lower total daily dose [11-13]. In addition, clinical outcome-based studies have shown that EI pip/tazo significantly lowers 14-day mortality rates and median hospital length of stays compared to patients receiving standard-infusion [14]. Rationale for use of extended-infusion therapy is also pharmacoeconomically sound. Hospitals save on drug acquisition costs due to the decrease in total daily dose.

Evidence supporting the efficacy and cost-effectiveness of EI pip/tazo has been clearly demonstrated. The following study investigated the possibility of a lower incidence of AKI with the extended-infusion formulation. The primary outcome of the study was to determine which administration method would have a higher incidence of AKI in combination with vancomycin. The secondary outcome was to identify potential risk factors for developing AKI.

\section{Materials and Methods}

This study was granted approval and exempt certification (SEH 9/2013-019) by the St. Elizabeth Institutional Review Board on November 5th, 2013. This retrospective review compared two subsets of patients admitted into SEH (St. Elizabeth Healthcare) Edgewood during two different 6-month periods: July to December, 2012 for the combination of SI pip/tazo plus vancomycin and July to December, 2013 for the combination of EI pip/tazo plus vancomycin. All patients receiving combination therapy were identified using the hospital's electronic medical record system.

\subsection{Inclusion and Exclusion Criteria}

All non- ICU (intensive care unit) patients admitted to SEH Edgewood greater than 18 years old, who received combination pip/tazo and vancomycin for a minimum of $48 \mathrm{~h}$ were screened for inclusion in this study. Exclusion criteria encompassed the following: patients with a baseline $\mathrm{SCr}$ greater than $2 \mathrm{mg} / \mathrm{dL}$, receiving peritoneal dialysis or hemodialysis, an ICU stay, pregnancy, concomitant use of defined nephrotoxic agents, as well as, those who received both formulations of pip/tazo therapy due to IV access and compatibility issues (Fig. 1). In this study, nephrotoxic agents comprised of aminoglycosides, IV contrast dye, amphotericin B, cyclosporine, tacrolimus, or vasopressors. Please note that the EI pip/tazo plus vancomycin group included patients who initially received one dose of SI pip/tazo in the emergency room, but were subsequently switched to EI pip/tazo upon admission to the hospital floor.

\subsection{Data Collection}

For the purpose of this review, acute kidney injury was defined as an absolute increase in $\mathrm{SCr}$ of 0.5 $\mathrm{mg} / \mathrm{dL}$ or a $50 \%$ increase from baseline. Data collected on all patients if available, included: baseline demographics of age, gender, BMI; use of ACE-I (angiotensin-converting enzyme inhibitors), angiotensin receptor blockers, thiazide diuretics, loop diuretics and NSAIDs; vancomycin trough levels; baseline $\mathrm{SCr}$ and the highest $\mathrm{SCr}$ during treatment or up to $72 \mathrm{~h}$ after completion of specified antibiotic therapy. 


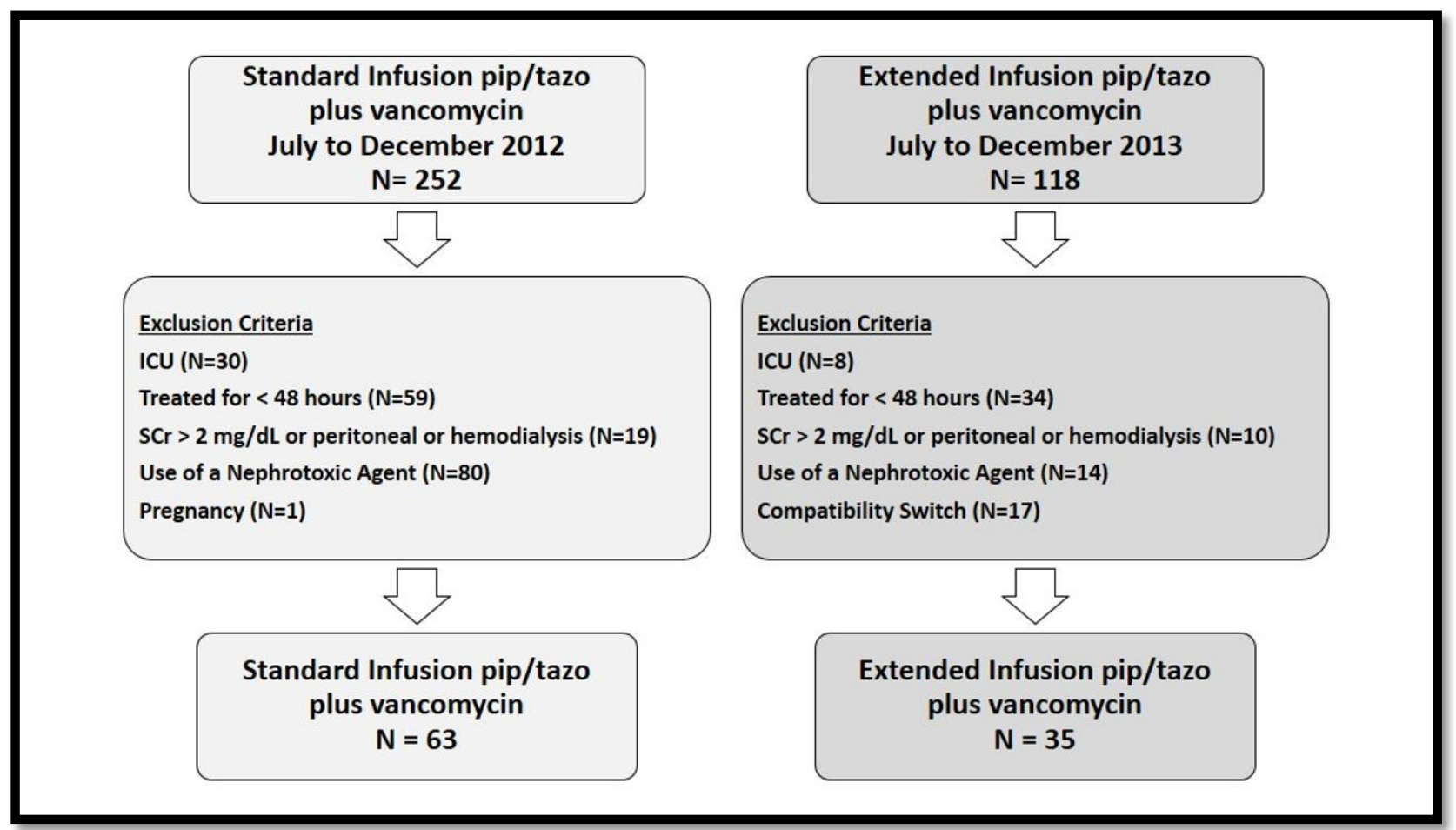

Fig. 1 A detailed view of the study design providing specific numbers of patients excluded per exclusion parameter. Nephrotoxic agents comprised of aminoglycosides, IV contrast dye, amphotericin B, cyclosporine, tacrolimus, or vasopressors. Abbreviations defined: pip/tazo = piperacillin/tazobactam, ICU = intensive care unit, $\mathrm{SCr}=$ serum creatinine.

\subsection{Statistical Analysis}

Statistical analysis was conducted by Burkhardt Consulting Center affiliated with the Northern Kentucky University Statistics Department. A Z-test on two proportions was used to compare the percent incidence of AKI between the two types of infusions. Binary logistic regression was used to identify independent variables that have an effect on the likeliness of AKI. Once an independent variable was identified as having a significant effect on the likeliness of AKI, a reduced binary logistic regression was then conducted to see if that independent variable had a significant interaction with the type of infusion. A Chi Square Test was then used to establish association between variables. All statistics utilized a $p$-value = 0.05 for statistical significance and $95 \%$ confidence interval.

\subsection{Study Population}

A total of 370 non-ICU patients greater than 18 years old receiving combination therapy for greater than 48 hours were screened for inclusion into the study. Exclusion criteria were applied to a total of 252 patients in the SI pip/tazo plus vancomycin group, leaving 63 patients and also applied to a total of 118 patients in the EI pip/tazo plus vancomycin group, leaving 35 patients to be included into the retrospective review. Fig. 1 shows a comprehensive breakdown of the exclusion criteria.

\section{Results}

A total of 98 patients receiving combination therapy were enrolled in this study, and their baseline demographics are available in Table 1 . The primary outcome of AKI occurred in 18 out of 63 patients $(28.6 \%)$ in the SI pip/tazo plus vancomycin group and 9 out of 35 patients $(25.7 \%)$ in the EI pip/tazo plus vancomycin group $(Z=-0.30, p$-value $=0.381)$. There was no statistically significant difference in the incidence of AKI between the two infusion types. The secondary outcome evaluated potential risk factors for 
Table 1 Baseline demographics for patients included in this retrospective review

\begin{tabular}{llll}
\hline Demographic & SI pip/tazo plus vancomycin & EI pip/tazo plus vancomycin & Total study population \\
& $N=63$ & $N=35$ & $N=98$ \\
\hline Male (\%) & $N=38(60)$ & $N=26(74)$ & $N=64(65)$ \\
Female (\%) & $N=25(40)$ & $N=9(26)$ & $N=34(35)$ \\
Age (mean \pm STD) & $63.1 \pm 20.5$ & $63.0 \pm 17.9$ & $63.1 \pm 19.4$ \\
BMI (mean \pm STD) & $29.5 \pm 8.6$ & $29.9 \pm 6.8$ & $29.6 \pm 7.9$ \\
\hline
\end{tabular}

SI = standard-infusion; EI = extended-infusion; pip/tazo = piperacillin/tazobactam; STD = standard deviation.

the development of AKI. In general, use of an ACE-I was identified as a significant risk factor for the development of AKI ( $p$-value $=0.05)$. In addition, there was a significantly higher incidence of AKI for patient's on an ACE-I and the standard infusion pip/tazo plus vancomycin combination $(p$-value $=0.034)$.

\section{Discussion}

Since research studies began to identify the increased risk of AKI with the combination of SI pip/tazo and vancomycin, pharmacists have begun to monitor patients' renal function and antibiotic levels more closely. As hospitals began to transition away from 30 minutes SI pip/tazo to 4 hours EI pip/tazo due to evidence supporting the efficacy and cost-effectiveness, questions were raised regarding a possible decrease of the incidence of AKI in general and with vancomycin. This study investigated the possibility of a lower incidence of AKI with the extended-infusion formulation in combination with vancomycin, in an effort to validate extended infusion from a safety perspective. While the study found no significant difference between the two administration methods, as pharmacists, we learned it is still necessary to closely monitor patients renal function by following trends in SCr, I \& O's (input and output levels), and vancomycin trough levels. Concomitant use of ACE-I's with this antibiotic combination may be a clinically relevant risk factor for the development of AKI; however with this limited sample size and study design it is hard to establish a definitive relationship.

\subsection{Limitations}

This study was limited by its retrospective design in that it cannot determine cause and effect. In addition, the lack of randomization and blinding made it difficult to control confounding variables and bias. A retrospective review of electronic medical records was relied on for accuracy and limited the amount of data collected. The study was a single-center study only conducted at SEH Edgewood and as a pharmacy resident research project, the one-year time constraint limited the sample size.

\subsection{Future Research}

This retrospective review is hopefully just the beginning of research that can still be done on this significant antibiotic-induced adverse event. Future research should be done prospectively at multiple facilities with larger sample sizes and further evaluation of additional risk factors. Other parameters that could be considered are the time to resolution of AKI and the need for renal replacement therapy. This study excluded all intensive care unit patients in an effort to eliminate the numerous confounding variables that exist in critically ill patients; however, it would be interesting to evaluate the incidence of AKI with this antibiotic combination in ICU patients versus general medical floor patients. Another possibility for future research would be to remove vancomycin from the study completely and specifically to evaluate SI pip/tazo versus EI pip/tazo for the incidence of AKI.

\section{Conclusions}

There was no statistically significant difference in the incidence of AKI between the two infusion types of piperacillin/tazobactam with vancomycin. However, AKI still remains very prevalent with one of our most 
commonly used empiric antibiotic combinations. While this study found no significant difference, as pharmacists, we learned it is still necessary to closely monitor patients' renal function by following trends in SCr, I \& O's, and vancomycin trough levels when patients are receiving this antibiotic combination, regardless of the method of administration.

\section{Acknowledgements}

This research received no specific grant from any funding agency in the public, commercial, or not-for-profit sectors. The authors have no actual or potential conflict of interest in relation to this original research article. The corresponding author would like to acknowledge Amanda Hayes, Pharm.D., for assisting in data collection.

\section{References}

[1] Dager, W., and Halilovic, J. 2014. "Acute Kidney Injury." In Pharmacotherapy: A Pathophysiologic Approach, edited by DiPiro, J. T., Talbert, R. L., Yee, G. C., Matzke, G. R., Wells, B. G., and Posey, L. M., 9th ed., New York: McGraw-Hill.

[2] Nolin, T. D., and Himmelfarb, J. 2014. "Drug-Induced Kidney Disease" In Pharmacotherapy: A Pathophysiologic Approach, edited by DiPiro, J. T., Talbert, R. L., Yee, G. C., Matzke, G. R., Wells, B. G., and Posey, L. M., 8th ed., New York: McGraw-Hill.

[3] Pannu, N., and Nadim, M. K. 2008. "An Overview of Drug-induced Kidney Injury.” Crit. Care Med. 36: 216-23.

[4] Meaney, C. J., Hynicka, L. M., and Tsoukleris, M. G. 2011. "Vancomycin-Associated Nephrotoxicity in Adult Medicine Patients: Incidence, Outcomes, and Risk Factors." Pharmacotherapy 34 (7): 653-61.

[5] Min, E., Box, K., Lane, J, Sanchez, J., Coimbra, R., Doucet, J., Potenza, B., and Wargel, L. "Acute Kidney Injury in Patients Receiving Concomitant Vancomycin and Piperacillin/Tazobactam." Critical Care Medicine 39 (12): (2011) 200.

[6] Hellwig, T., Hammerquist, R., Loecker, B., and Shields, J. 2011. "Retrospective Evaluation of the Incidence of
Vancomycin and/or Piperacillin-Tazobactam Induced Acute Renal Failure.” Critical Care Medicine 39 (12): 79.

[7] Pritchard, L., Baker, C., Leggett, J., Sehdev, P., Brown, A., and Bayley, K. B. 2010. "Increasing Vancomycin Serum trough Concentrations and Incidence of Nephrotoxicity." Am. J. of Med. 123 (12): 1143-9.

[8] Jeffres, M. N., Isakow, W., Doherty, J. A., Micek, S. T., and Kollef, M. H. 2007. "A Retrospective Analysis of Possible Renal Toxicity Associated with Vancomycin in Patients with Health Care-Associated Methicillin-Resistant Staphylococcus Aureus Pneumonia." Clin. Ther. 29 (6): 1107-15.

[9] Jensen, J. S., Lars, H., Lundgren, B., Bestle, M. H., Mohr, T., Andersen, M. H., Thornberg, K. J., Løken, J., Steensen, M., Fox, Z., Tousi, H., Søe-Jensen, P., Lauritsen, A. Ø., Strange, D. G., Reiter, N., Thormar, K., Fjeldborg, P. C., Larsen, K. M., Drenck, N. E., Johansen, M. E., Nielsen, L. R., Ostergaard, C., Kjær, J., Grarup, J., and Lundgren, J. D. 2012. "Kidney Failure Related to Broad-Spectrum Antibiotics in Critically Ill Patients: Secondary End Point Results from a 1200 Patient Randomized Trial." BMJ Open 2 (2): 1-8.

[10] Moenster, R. P., Linneman, T. W., Finnegan, P. M., Hand, S., Thomas, Z., and McDonald, J. R. 2014 “Acute Renal Failure Associated with Vancomycin and Beta-lactams for the Treatment of Osteomyelitis in Diabetics: Piperacillin-Tazobactam as Compared with Cefepime." Clinical Microbiology and Infection 20 (6): 384-9.

[11] Ambrose, P. G., Bhavnani, S. M., Rubino, C. M., Louie, A., Gumbo, T., Forrest, A., and Drusano, G. L. 2007. "Pharmacokinetics-Pharmacodynamics of Antimicrobial Therapy: It's not Just for Mice Anymore." Clin. Infect. Dis. 44: 79-86.

[12] Craig, W. A. 1998. "Pharmacokinetic/Pharmacodynamic Parameters: Rationale for Antibacterial Dosing of Mice and Men." Clin. Infect. Dis. 26: 1-10.

[13] Lodise, T. P., Lomaestro, B. M., and Drusano, G. L. 2006. "Application of Antimicrobial Pharmacodynamic Concepts into Clinical Practice: Focus on Beta-lactam Antibiotics: Insights from the Society of Infectious Diseases Pharmacists." Pharmacotherapy 26: 1320-32.

[14] Lodise, T. P., Lomaestro, B., and Drusano, G. L. 2007. "Piperacillin-Tazobactam for Pseudomonas Aeruginosa Infection: Clinical Implications of an Extended-Infusion Dosing Strategy." Clin. Infect. Dis. 44: 357-63. 